\title{
A PRÁTICA DAS EVIDÊNCIAS DOCUMENTAIS NA ATIVIDADE DE AUDITORÍA ${ }^{1}$
}

\author{
LA PRÁCTICA DE LA EVIDENCIA DOCUMENTAL EN EL ENCARGO \\ DE LA AUDITORÍA
}

THE PRACTICE OF DOCUMENTARY EVIDENCE IN AUDITING

Recebido: 16/11/2017 - Aprovado: 14/03/2018 - Publicado: 26/06/2018 Processo de Avaliação: Double Blind Review

\author{
Ludivia Hernandez Aros ${ }^{2}$ \\ Magister Internacional en Auditoria y gestión Empresarial \\ Universidad Cooperativa de Colombia \\ Ludivia.Hernandez@campusucc.edu.com \\ Laura Gallego Cossio \\ Magister en Finanzas Corporativas \\ Universidad Cooperativa de Colombia \\ laura.gallego@campusucc.edu.co \\ Yury Enid Riveros Marín \\ Estudiante de Contaduría Pública. \\ Universidad Cooperativa de Colombia \\ yury.riveros@campusucc.edu.co

\section{Edwar Dirley Oyola Bejarano} \\ Estudiante de Contaduría Pública. \\ Universidad Cooperativa de Colombia \\ edwar.oyolab@campusucc.edu.co
}

RESUMEN: El presente artículo analiza la importancia que tiene la obtención, presentación y estudio de la evidencia documental como prueba suficiente y relevante en el encargo de auditoría. Este estudio se soporta en la conceptualización e información teórica sobre las

\footnotetext{
${ }^{1}$ Artículo derivado de proyecto de investigación asociado al Grupo PLANAUDI, adscrito al Centro de Investigaciones del programa de Contaduría Pública - Línea de Investigación Control y Aseguramiento de la Universidad Cooperativa de Colombia, sede Ibagué - Cl. 10 \#64, Ibagué-Tolima, Colombia, financiado el mismo por CONADI

${ }^{2}$ Autor para correspondência: Calle 10 No. 1 - 120 EdificioUrrutia. Ibagué. Colombia.
}

REMIPE- Revista de Micro e Pequenas Empresas e Empreendedorismo da Fatec Osasco 
características de un documento válido en el encargo de auditoría, generalidades de los documentos y la importancia de los mismos como prueba de evidencia documental. Metodológicamente el presente estudio es de corte descriptivo, parte de un enfoque cualitativo por basarse en un estudio de información sin medición numérica, utilizando el método analítico. Los resultados de la investigación confirman que la práctica de la evidencia documental-la cual es obtenida a través de la inspección física o electrónica de los documentos que soportan las operaciones y las transacciones- genera mayor confianza y credibilidad en el proceso auditor en lo que respecta al trabajo de campo, debido a que proporciona los elementos necesarios para que el ejercicio de auditoría sea confiable, productivo y a su vez pueda generar valor agregado a la organización objeto de auditoría, convirtiéndose en acciones de mejoramiento y garantía para la empresa y la comunidad.

Palabras clave: Evidencia documental, Riesgo de auditoría, Evidencia relevante, Encargo de auditoría, Proceso Judicial.

RESUMO: Este artigo analisa a importância em obter, apresentar e estudar as provas documentais como evidência suficiente e relevante na tarefa de auditoria. Este estudo é apoiado em pesquisa bibliográfica sobre as características dos documentos válidos nas tarefas de auditoria, generalidades dos documentos e sua importância como prova de evidência documental. Metodologicamente, o presente estudo é descritivo, parte de uma abordagem qualitativa baseada em um estudo de informação sem mensuração numérica, utilizando o método analítico. Os resultados da pesquisa confirmam que a prática da prova documental, obtida por meio da inspeção física ou eletrônica dos documentos que suportam operações e transações, gera maior confiança e credibilidade no processo de auditoria, em relação ao trabalho de campo. Este fato ocorre por fornecer os elementos necessários para que o exercício de auditoria seja confiável, produtivo, e, por sua vez, para que se possa gerar valor agregado à organização objeto de auditoria, tornando-se em ações de melhoria, de forma a possibilitar garantias para a empresa e para a comunidade.

Palavras-chave: Evidência documental, Risco de auditoria, Evidências relevantes, Comissão de auditoria, Processo judicial. 
ABSTRACT: This article shows the importance of obtaining, presenting and analyzing documentary evidence as sufficient and relevant evidence in the audit engagement. This study is supported in the conceptualization and theoretical information about the characteristics of a valid document in the audit assignment, generalities of the documents and the importance of the same as evidence of documentary evidence. Methodologically the present study is descriptive, part of a qualitative approach to be based on an information study without numerical measurement, using the analytical method. The results of the investigation confirm that the practice of documentary evidence - which is obtained through the physical or electronic inspection of the documents that support operations and transactions - generates greater confidence and credibility in the audit process as regards To the field work, because it provides the necessary elements for the audit exercise to be reliable, productive and in turn can generate added value to the organization being audited, becoming actions of improvement and guarantee for the company and the community.

Keywords: Documentary Evidence, Audit risk, Relevant evidence, Audit engagement, Judicial process

\section{INTRODUCCIÓN}

La evidencia en auditoría se define como la convicción razonable que tiene el auditor, de que las cifras contables incluidas en las cuentas anuales son el resultado de los acontecimientos económicos que realmente han ocurrido durante el periodo de tiempo al que se refieren aquellas, y que se encuentran debidamente soportados por el sistema de información contable (Rodriguez B. , 2015).

Partiendo de que la evidencia se puede clasificar en evidencia física, documental,informática, testimonial o analítica, el presente artículo pretende abarcar aspectos generales en lo que respecta a la práctica de la evidencia documental en el encargo de auditoría.

En términos simples, la evidencia documental es un tipo de evidencia que abarca varios registros que dan soporte al negocio de la empresa y al sistema de información contable: cheques, facturas, contratos y minutas de las juntas(Zambrana, 2012). Cabe aclarar 
que, en gran parte, la confiabilidad de ésta evidencia depende de si fue preparada dentro de la empresa o fuera de ella, dado que algunos documentos creados en la compañía se envían fuera de ella para que sean procesados y gracias a esta revisión crítica de externos, se consideran más confiables que otros documentos elaborados por el personal del ente auditado.

Tal y como se desprende de la anterior definición, las evidencias externas abarcan, entre otras, cartas, facturas de proveedores, contratos, auditorías externas y otros informes o dictámenes y confirmaciones de terceros, mientras que las evidencias internas tienen su origen en la organización, lo cual incluye, entre otros, registros contables, correspondencias enviadas, descripciones de puestos de trabajo, planes, presupuestos, informes internos, políticas y procedimientos internos.

En efecto, la evidencia documental es de suma importancia dentro del encargo de auditoría debido a que los hechos encontrados en el proceso de auditoría deben estar suficientemente respaldados por evidencias, documentos y pruebas que garanticen la veracidad de lo que se está reportando e informando. Así las cosas, el auditor debe obtener sólo las evidencias que respondan a los objetivos específicos de la auditoría, que son los de averiguar si los estados financieros son completos, auténticos, razonables y uniformes. Por tanto, cualquier evidencia que se aparte de la consecución de estos objetivos no deberá ser tenida en cuenta(IBEF, 2012).

\section{EL DOCUMENTO COMO EVIDENCIA DOCUMENTAL}

Vesper (2009), considera que el documento es una forma organizada de información que puede servir como evidencia. Esta información puede estar en muchas clases de formato, soporte o medio, tales como papel, audio, informático o visual; así mismo, de acuerdo a la Unión Francesa de Organismos de Documentación, el documento se define como "todo elemento de conocimiento o fuente de información registrada, materialmente susceptible de ser utilizada para consulta, estudio o prueba"(Yepes, 1993,p.36).Concomitante con lo anterior, los documentos conforman una prueba documental debido a que gozan de una presunción de legitimidad, autenticidad y veracidad, sin embargo, esa presunción puede ser desvirtuada mediante prueba en lo contrario(Cruz Navas et al., 2011).

Ahora bien, en materia contable, los documentosson los soportesque sirven de base para registrar las operaciones comerciales en una empresa, estos soportes se elaboran en 
original y tantas copias como las necesidades de la empresa lo exijan. Su objetivo es controlar las operaciones practicadas y la comprobación de los asientos contables con el fin demantener un apropiado control de todas las acciones que se realizan dentro del ente económico. En efecto, el Código de Comercio, su artículo 48, establece en que todo comerciante deberá conformar su contabilidad, libros, registros contables, inventarios y estados financieros en general, según las disposiciones del mismo(Rodriguez J. G., 2011).

El documentose presenta como prueba -evidencia documental-, dentrodel encargo de auditoría, debido a que estos son los únicos soportes con los que se cuenta para que el auditor pueda basar su opinión y emitir un informe razonable sobre la realidad económica de la organi zación auditada.

\subsection{CARACTERÍSTICAS DE UN DOCUMENTO VÁLIDO EN EL ENCARGO DE AUDITORIA}

El propósito colectivo de todos los procedimientos de auditoría es el de obtener evidencia para formarse una opinión sobre la imparcialidad de los estados financieros en conjunto(Glezen, 1997), en este estudio, la evidencia documental es uno de los tipos de evidencia más importantes que utiliza el auditor, y en el cual se apoya con mayor o menor intensidad dependiendo de la confianza que le ofrezca el sistema de control interno de la empresa.

Los resultados del auditor se ven reflejados en el informe final, que es respaldado por las evidencias recolectadas - en la ejecución de su trabajo-, como documentos u otro tipo de pruebas, que soporte su informe Trujillo (1990), Establece que Debe obtenerse evidencia válida y suficiente por medio de análisis, inspección, observación, interrogación, confirmación y otros procedimientos de auditoría, con el propósito de allegar bases razonables para el otorgamiento de un dictamen sobre los Estados Financieros sujetos a revisión.

El siguiente gráfico presenta las generalidades de los documentos y las características que deben cumplir para que éstos sean considerados como válidos dentro del encargo de auditoría: 
Figura1: Generalidades de los documentos:

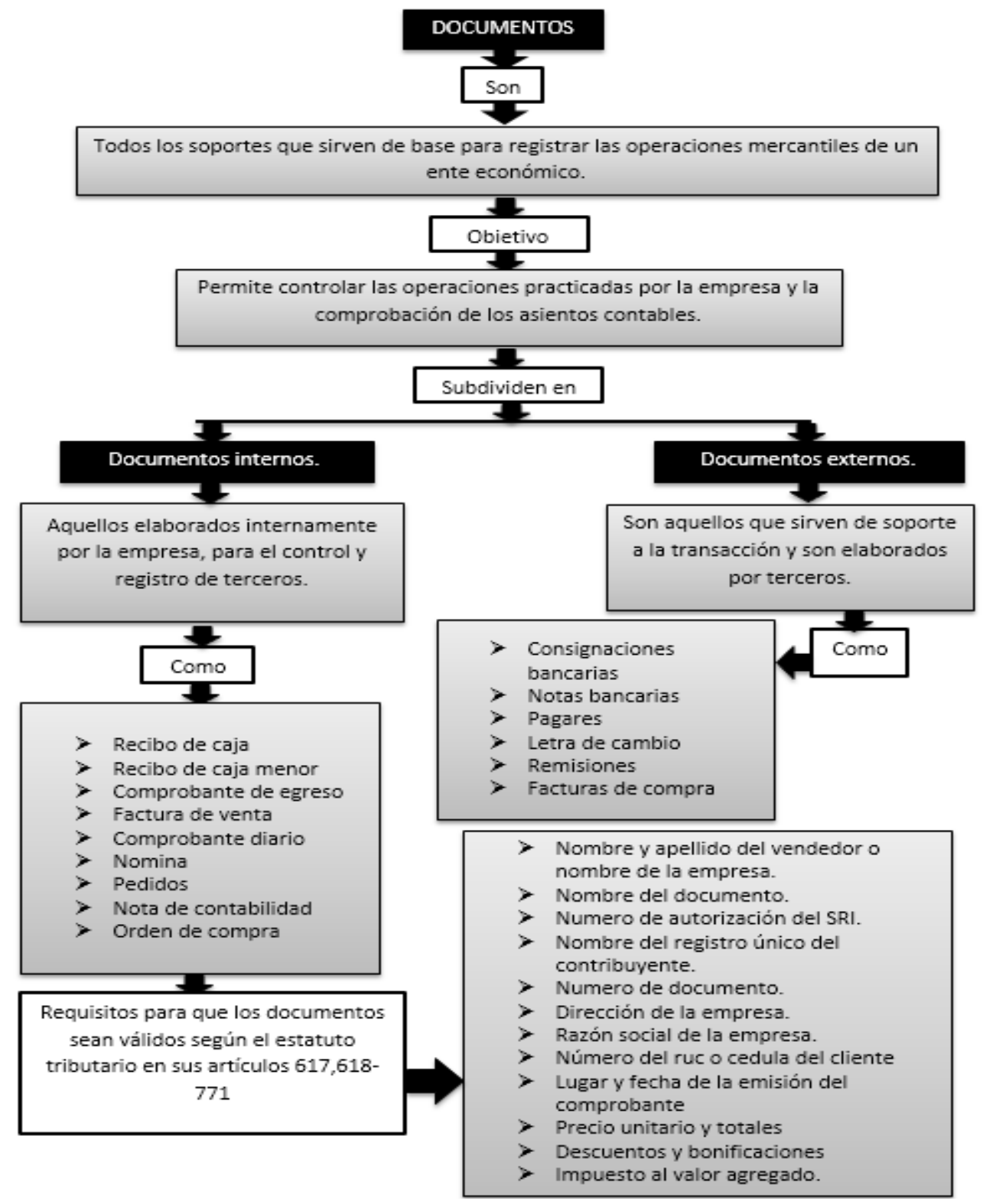

Fuente: Elaboración Propia.

Así pues, se deben mantener registros de los procedimientos aplicados como pruebas desarrolladas, información obtenida y conclusiones pertinentes a que se llegó en eltrabajo, y de la misma manera el contador debe preparar en su encargo de auditoria documentos como: a) programas de auditoría; cuestionarios y hojas de puntos pendientes de investigar; hojas de trabajo relativas a los saldos de cuentas de control y auxiliares; b) hojas de trabajo con los resultados de las pruebas de cumplimiento; c) cuestionarios con evaluación de los controles; 
asientos de ajustes y de reclasificaciones; d) planillas, hojas de análisis y otros papeles de soporte; e) extractos de actas y otros papeles narrativos; f) certificaciones y confirmaciones; escrituras de constitución y reformas; y g) borradores del dictamen de los estados financieros y de las notas con el propósito de dar justificación y soporte al trabajo efectuado.

\subsection{IMPORTANCIA DE LA EVIDENCIA DOCUMENTAL;}

Evidencia en auditoria es toda la información que usa el auditor para llegar a las conclusiones a partir de las cuales basa la opinión de auditoría, e incluye los registros de contabilidad subyacentes a los estados financieros y otra información. Si bien, la confiabilidad de la evidencia de auditoría está influenciada por su fuente y por su naturaleza y depende de las circunstancias individuales bajo las cuales se obtiene(Luna, 2004).

En consecuencia,la evidencia y los papeles de trabajo constituyen el soporte fundamental de los hallazgos detectados por el auditor, de ahí la importancia que reviste la suficiencia,que significa la cantidad de la evidencia, y su relevancia, es decir la calidad de la misma.Así como la calidad y claridad de los papeles de trabajo, atendiendo a que la información de aquí se recoge está escrita siempre a terceros que son los clientes del servicio de auditoría(Bautista, 2013).

Visto así, la evidencia es la convicción que tiene que tener el auditor de que los datos contables recogidos en las cuentas anuales están perfectamente soportados en el tiempo, y ésta se debe obtener a través del resultado de las pruebas de auditoría aplicadas según las circunstancias de cada caso y de acuerdo con el juicio profesional del auditor.

La siguiente figura presenta las generalidades de la evidencia dentro de la auditoría: 
Figura2 - Generalidades de la evidencia:

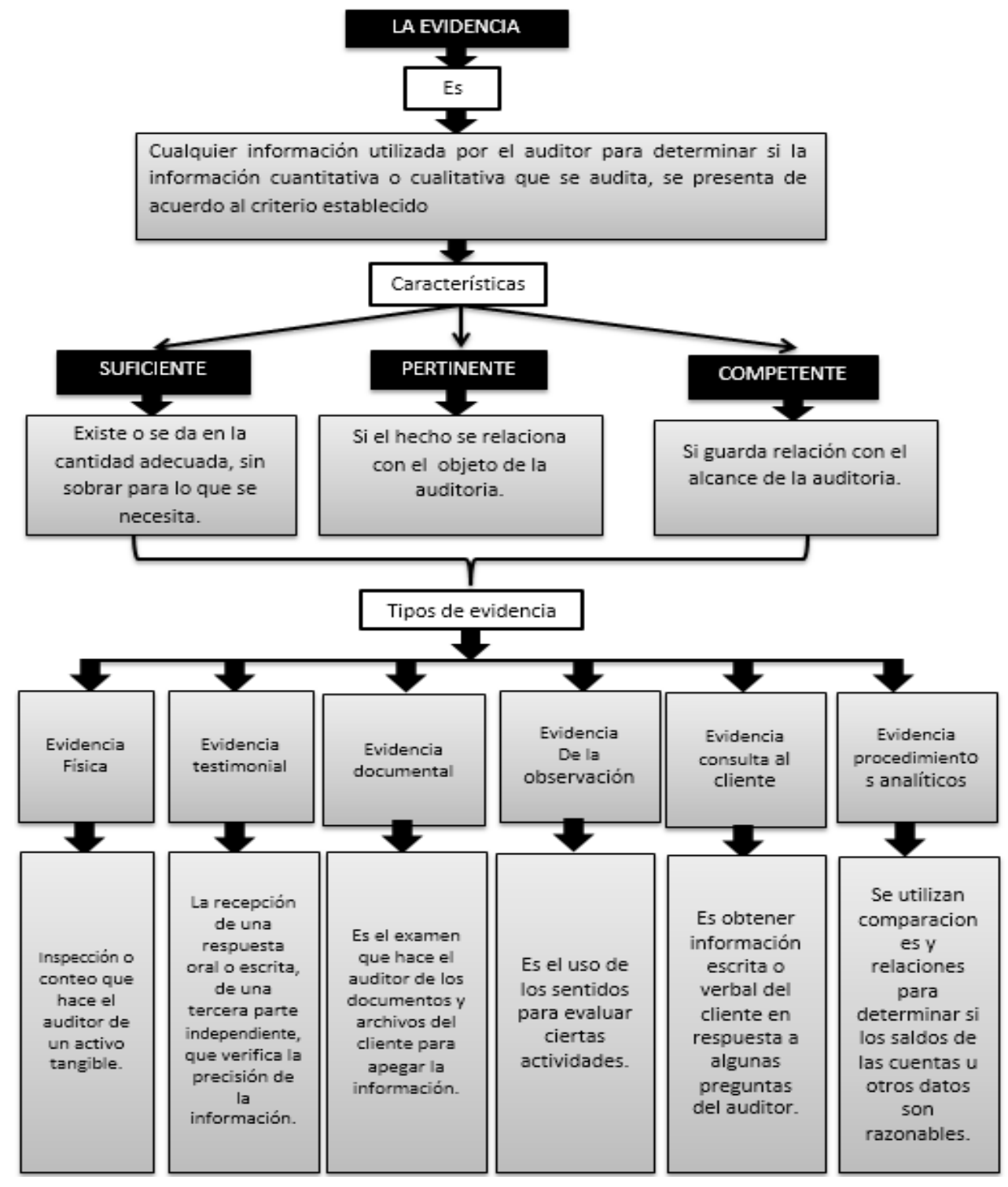

Fuente: Elaboración Propia

En ese caso, la recolección de la evidencia documental, según el Instituto Barcelona de Estudios Financieros, necesita de un conocimiento profundo de la empresa y de todas las transacciones que ésta origina, debido a que sus fuentes deben abarcar desde los sistemas contables y la documentación en que se fundamentan, hasta el contacto con los clientes, proveedores y otros terceros que, de alguna forma, operan con la empresa o tienen conocimiento de sus actividades(IBEF, 2012). 
Es importante que el auditor aprecie las diferentes características de la evidencia deauditoría y la confianza que se puede depositar en cada clase de evidencia.Por tanto, la evidencia en auditoria se puede caracterizar como: La generada por el cliente y que éste tiene en su poder; la recibida de fuentes externas y que está en poder del cliente; y la determinada recibida directamente por el auditor recurriendo a medios independientes o que proviene de fuentes independientes o casi independientes(Glezen, 1997).

Teniendo en cuenta las características anteriormente mencionadas, la fiabilidad e integridad de la evidencia está en relación con la fuente de la que se obtenga interna y externamente, y también con su naturaleza, es decir, visual, documental y oral.

No obstante, aunque la fiabilidad depende de las circunstancias en las que se obtiene, la evidencia documental puede evaluarse tomando en consideración los siguientes factores: a) La evidencia externa es más fiable que la interna; b)La evidencia interna es más fiable cuando los controles internos relacionados con ellos son satisfactorios; c) La evidencia obtenida físicamente por el propio auditor mediante un examen, observación, cálculo o inspección es más confiable que la que la obtenida por la empresa, y d) La evidencia en forma de documentos y manifestaciones escritas es más fiable que la procedente de declaraciones orales.

Por tanto, la evidencia documental que soporta la elaboración de los estados financieros está conformada por información contable que registra los hechos económicos y por los demás datos que corrobore esta última, todo lo cual deberá estar disponible para el examen del revisor fiscal o auditor externo(Decreto 2420, 2015).

Los libros oficiales, los auxiliares, los manuales de procedimientos y contabilidad, los papeles de trabajo, los justificantes de los comprobantes de diario, las conciliaciones, etc., constituyen evidencia documental primaria que apoya la preparación de los estados financieros(Normas Relativas a la Ejecucion del Trabajo, 2003). Sin embargo, los datos de contabilidad que se utilizan en la elaboración de los estadosno pueden ser considerados suficientes por sí mismos y, por otra parte, no puede justificarse una opinión sobre tales estados sin la comprobación de la corrección y exactitud de los datos básicos.

Para ello se utiliza la evidencia comprobatoria, en la cual se incluyen materiales documentados como, cheques, facturas, contratos, actas, confirmaciones y otras declaraciones escritas por personas responsables, lo cual permite que el auditor o revisor fiscal obtenga 
información por medio de su investigación, observación, inspección y examen físico con el fin de llegar a ciertas conclusiones a través de razonamiento lógico.

Es de aclarar que los documentos pertinentes para respaldar asientos en las cuentas y representaciones en los estados financieros ordinariamente están a la mano en los archivos de la compañía y pueden ser utilizados por el auditor para su examen. De igual forma, dentro de la organización y fuera de ella, existen también personas informadas a quienes el auditor puede dirigir sus preguntas.En definitiva,la NIA 500, contiene las fases relevantes y procedimientos de cumplimiento para la obtención de la cantidad y calidad de la evidencia de auditoría que se debe obtener cuando se auditan los estados financieros. Esta Norma Internacional de Auditoría explica lo que constituye la evidencia en una auditoría de estados financieros, y trata de la responsabilidad que tiene el auditor de diseñar y aplicar procedimientos de auditoría para obtener evidencia de auditoría suficiente y adecuada que le permita alcanzar conclusiones razonables en las que basar su opinión. Es por esta razón que cuando se aplique esta NIA a determinada auditoria se debe cumplir con su propósito y finalidad para obtener excelentes resultados que permitan a la empresa tomar buenas decisiones y así cumplir con su misión(Medina, 2014).

\section{METODOLOGÍA}

El presente artículo de corte descriptivoparte de un enfoque cualitativo por basarse en un estudio de información sin medición numérica, utilizando el método analíticoapoyado en la revisión, reflexión y análisis del marco teórico en elcuase encuentran conceptos propios de auditoría vistos desde la normatividad internacional, buscando el deber ser del documento dentro del proceso realizado por el auditor en una organización, para luego relacionar estos conceptos con la práctica de la evidencia documental en el encargo de auditoría, generando resultados que permitan identificar y valorar los riesgos yestablecer procesos para la obtención, análisis y presentación de la evidencia teniendo en cuenta características propias de las normas internacionales de auditoria en cuanto a la planeación, la ejecución y su posterior reconocimiento en el informe oficial emitido por el auditor.

Para el cumplimiento y el buen desarrollo de los objetivos propuestos se plantearon las siguientes fases:

- $\quad$ Fase 1:Documentación, identificación y valoración de riesgos en la obtención 
de la evidencia documental a través de la elaboración de una matriz de riesgos.

- $\quad$ Fase 2:Conceptualización y análisisdel procedimiento para la obtención, análisis y presentación de la evidencia documental por medio de un diagrama de procesos.

- $\quad$ Fase 3: Valoración de la evidencia documental del perito contable en el proceso judicial mediante un estudio de caso.

\section{RESULTADOS}

La auditoría es "un proceso sistemático, practicado por los auditores de conformidad con normas y procedimientos técnicos establecidos, consistente en obtener y evaluar objetivamente las evidencias sobre las afirmaciones contenidas en los actos jurídicos o eventos de carácter técnico, económico, administrativo y otros, con el fin de determinar el grado de correspondencia entre esas afirmaciones, las disposiciones legales vigentes y los criterios establecidos"(Sandovalesco, 2013), ésta debe funcionar como una actividad que tenga el fin de agregar valor y mejorar las operaciones en una organización, así como contribuir al cumplimiento de sus objetivos y metas; aportando un enfoque que permita evaluar y mejorar la eficacia de los procesos de gestión de riesgos, control y dirección.

Así las cosas, el auditor debe considerar la importancia relativa y las relaciones con el riesgo del trabajo desde el momento que planea y desarrolla un servicio de auditoría, con el propósito de reducir el riesgo de expresar una conclusión inapropiada. Para ello, el auditor deberá documentar los asuntos que son importantes para apoyar las conclusiones expresadas en el informe de auditoría y a su vez, dejar evidencia de que la auditoría se llevó a cabo de acuerdo con las normas técnicas de trabajo señaladas por los organismos profesionales.

Tal documentación significa el material, papeles de trabajo preparados, obtenidos o retenidos por el auditor en conexión con la ejecución de la auditoría, los cuales pueden estar presentados en diversas formas o a través de diferentes medios. Por consiguiente, el auditor planea y realiza el trabajo de manera tal que reduzca a un nivel aceptable el riesgo de expresar una conclusión inapropiada; así pues, se debe tener en cuenta que los riesgos pueden ser inherentes, los cuales tienen que ver exclusivamente con la actividad económica de la empresa; de control que se presentan cuando los controles no existen u operan inefectivamente, y de detección que son los relacionados con los procedimientos de auditoría por lo que se trata de la no detección de la existencia de error en el proceso realizado. 
En síntesis, la evidencia documental en el encargo de auditoría comprenderá documentos fuente y registros contables, información corroborativa de otras fuentes, procedimientos sobre el manejo de las áreas o divisiones e indicadores de gestión. Lo cual se obtiene de una aplicación apropiada de pruebas de control, de procedimientos sustantivos, análisis de proyecciones y análisis de los indicadores claves de éxito, utilizados por el auditor para satisfacerse sobre la legitimidad de la información, haciendo las comprobaciones necesarias para hacerse un juicio profesional.

\subsection{VALORACIÓN DE LOS RIEGOS DEL AUDITOR EN LA OBTENCIÓN DE LA EVIDENCIA DOCUMENTAL}

De acuerdo con lo que establece la NIA 200 "Objetivos y Principios que Gobiernan la Auditoria de Estados Financieros", cuando un auditor independiente emite una opinión acerca de la razonabilidad de los estados financieros de una entidad, éste siempre se enfrentará a laposibilidad de que su opinión sea inapropiada. A esta probabilidad de error se le conoce como "riesgo de auditoría".

De igual forma, se entiendecomo riesgo probable la posibilidad de que el auditor no detecte un error significativo que pudiera existir en las cuentas por falta de evidencia respecto a una determinada partida o por la obtención de una evidencia deficiente o incompleta sobre la misma. Para ello deben diseñarse y ejecutarse procedimientos de auditoría que resulten en un riesgo reducido y aceptable de que la opinión que se exprese sobre las cuentas anuales sea inadecuada. Sin lugar a dudas, y conforme a lo mencionado, es necesario tratar de manera singular la justificación documental suficientey hay que asegurarse de que las pruebas documentales o las justificaciones que amparan sus conclusiones son suficientemente lidas(Ferrer, 2012). En concordancia, el auditor como profesional capacitado y experimentado tiene que basar todos sus principios en habilidades y destrezas durante el desarrollo de sus funciones y así poner en práctica los conocimientos de temas esenciales que permitan viabilizar el trabajo en la auditoria. Según la ( NIIC 1, 2009) el auditor deberá establecer políticas y procedimientos diseñados para proporcionarle a la organización objeto de auditoría, una seguridad razonable de que los encargos se realizan de conformidad con las normas profesionales y con los requerimientos legales y aplicables, y de que se emite un informe que es adecuado en función de las circunstancias y basado en la evidencia 
documental obtenida y recopilada en los papeles de trabajo.No obstante, se debe tener en cuenta que al momento de aplicar técnicas y procedimientos de auditoría con el fin de obtener la evidencia documental se pueden presentar riesgos que dependen en gran parte de las fuentes de donde se obtiene tal evidencia.

De acuerdo lo anterior, se valorarán los riesgos en el proceso de obtención de la evidencia documental atendiendo a su clasificación y sus fuentes, a través de la siguiente matriz

Tabla 1. Matriz de valoración de riesgos en la obtención de la evidencia documental elaborada y retenida por la entidad:

\begin{tabular}{|c|c|c|c|c|c|c|c|}
\hline \multicolumn{3}{|c|}{ POCOFRECUENTE } & \multicolumn{2}{|l|}{ Probebtidad 1 } & \multicolumn{2}{|l|}{ BAIO } & Ninel de nesszo ! \\
\hline \multicolumn{3}{|c|}{ FRECUENTE } & \multicolumn{2}{|l|}{ Probeblidad 2} & \multicolumn{2}{|c|}{ MODERADO } & Ninel de resszo 2 \\
\hline \multicolumn{3}{|c|}{ MUY FRECUENIE } & \multicolumn{2}{|l|}{ Probeblided 3} & \multicolumn{2}{|c|}{ ALTO } & Nirel de nesgo 3 \\
\hline \multicolumn{8}{|c|}{ Jatriz De Valoración De Riesgos Del Auditor De La Obtención De La Evidencia Documental } \\
\hline $\begin{array}{l}\text { Tipo De } \\
\text { Erideacia }\end{array}$ & \multicolumn{2}{|c|}{ Tipo De Riesgo } & Descripción & $\mathrm{NIA}$ & Probabilidad & $\begin{array}{l}\text { Nirel De } \\
\text { Riesgo }\end{array}$ & Sugerencia \\
\hline \multirow{4}{*}{ 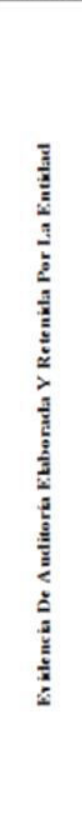 } & $\begin{array}{l}\text { Ruesgo de la } \\
\text { Auditoria }\end{array}$ & Intimidación & 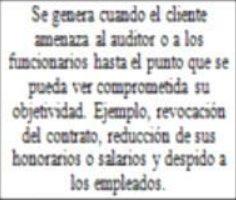 & 500 & Poco Frecueate & Atso & 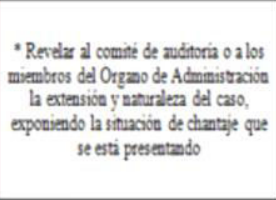 \\
\hline & $\begin{array}{c}\text { Riesgo del } \\
\text { Auditor }\end{array}$ & Propio Interés & 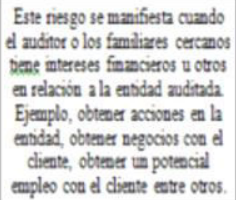 & 200 & Poco Frecuente & Ats & 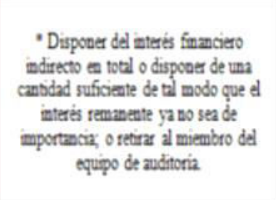 \\
\hline & $\begin{array}{l}\text { Riesgo } \\
\text { Humano }\end{array}$ & $\begin{array}{l}\text { Enrores del } \\
\text { Personal }\end{array}$ & 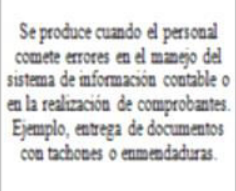 & 520 & Frecuente & Also & 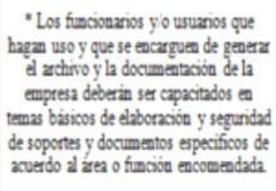 \\
\hline & Semiestro & Incendio o Robo & 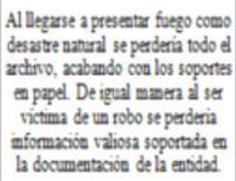 & 240 & Poco Frecueste & Alto & 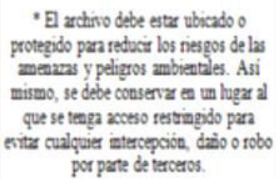 \\
\hline
\end{tabular}

Fuente: Elaboración propia 
Tabla 2. Matriz de valoración de riesgos en la obtención de la evidencia documental elaborada y renida por tercero:

\begin{tabular}{|c|c|c|c|c|c|c|c|}
\hline \multicolumn{3}{|c|}{ POCO FRECUENTE } & \multicolumn{2}{|l|}{ Probabilidad 1} & \multicolumn{2}{|c|}{ BAJO } & Nivel de riesgo 1 \\
\hline \multicolumn{3}{|c|}{ FRECUENTE } & \multicolumn{2}{|l|}{ Probabilidad 2} & \multicolumn{2}{|c|}{ MODERADO } & Nivel de riesgo 2 \\
\hline \multicolumn{3}{|c|}{ MUY FRECUENTE } & \multicolumn{2}{|l|}{ Probabilidad 3} & \multicolumn{2}{|c|}{ ALTO } & Nivel de riesgo 3 \\
\hline \multicolumn{8}{|c|}{ Matriz De Valoración De Riesgos Del Auditor De La Obtención De La Evidencia Documental } \\
\hline $\begin{array}{c}\text { Tipo De } \\
\text { Evidencia }\end{array}$ & \multicolumn{2}{|c|}{ Tipo De Riesgo } & Descripción & NIA & Probabilidad & $\begin{array}{c}\text { Nivel De } \\
\text { Riesgo }\end{array}$ & Sugerencia \\
\hline \multirow{4}{*}{ 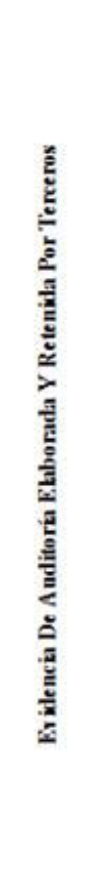 } & $\begin{array}{c}\text { Riesgo } \\
\text { De La } \\
\text { Auditoria }\end{array}$ & $\begin{array}{l}\text { Negación De } \\
\text { Información }\end{array}$ & $\begin{array}{l}\text { Se presenta cuando el tercero se } \\
\text { niega a suministrar información al } \\
\text { equipo auditor para proceder a la } \\
\text { confimación y corroboración de } \\
\text { transacciones, saldos, entre otros. }\end{array}$ & 315 & Poco Frecuente & Bajo & $\begin{array}{l}\text { *Enviar una segunda circularización } \\
\text { solicitando la información respectiva, en } \\
\text { caso de no ser aceptada ni remitida la } \\
\text { documentación solicitada se debe } \\
\text { descartar esta evidencia como prueba } \\
\text { suficiente y relevante. }\end{array}$ \\
\hline & $\begin{array}{l}\text { Riesgo } \\
\text { Del } \\
\text { Auditor }\end{array}$ & $\begin{array}{l}\text { Familiaridad } 0 \\
\text { Confianza }\end{array}$ & $\begin{array}{l}\text { Es generado cuando el auditor } \\
\text { tiene una relación cercana con el } \\
\text { tercero y puede ver comprometida } \\
\text { su objetividad. Ejemplo, una } \\
\text { relación directa o indirecta con } \\
\text { clientes, proveedores o } \\
\text { instituciones fimancieras, a quienes } \\
\text { se les solicita documentación. }\end{array}$ & 315 & Poco Frecuente & Alto & $\begin{array}{l}\text { - Estructurar las responsabilidades del } \\
\text { equipo auditor de modo que este } \\
\text { profesional no esté involucrado en } \\
\text { asumtos que esten a cargo de la persona } \\
\text { cercana o familiar. }\end{array}$ \\
\hline & $\begin{array}{l}\text { Riesgo } \\
\text { Humano }\end{array}$ & $\begin{array}{l}\text { Manipulación } \\
\text { De La } \\
\text { Información }\end{array}$ & $\begin{array}{l}\text { Este riesgo se da cuando la } \\
\text { evidencia que esti en poder del } \\
\text { tercero puede ser susceptible de } \\
\text { modificación y manipulacion para } \\
\text { beneficio de ambas partes }\end{array}$ & 315 & Frecuente & Alto & $\begin{array}{l}\text { * Inspeccionar que la empresa auditada } \\
\text { tenga copia de los soportes de las } \\
\text { transacciones realizadas con terceros para } \\
\text { evitar que se produzcan modificaciones } \\
\text { en los documentos. }\end{array}$ \\
\hline & Simiestro & $\begin{array}{l}\text { Perdida De } \\
\text { Archivo }\end{array}$ & $\begin{array}{l}\text { Se produce cuando los } \\
\text { documentos y soportes elaborados } \\
\text { por terceros pueden desaparecer } \\
\text { por falta de orden y control en las } \\
\text { prácticas y politicas de gestion } \\
\text { documental que implementa la } \\
\text { empresa }\end{array}$ & 230 & Frecuente & Blto & $\begin{array}{l}\text { *Verificar los documentos que se tienen } \\
\text { en poder del cliente y corroborar con los } \\
\text { registros en el sistema de información } \\
\text { contable del tercero }\end{array}$ \\
\hline
\end{tabular}

Fuente: Elaboración propia. 
Tabla 3. Matriz de valoración de riesgos en la obtención de la evidencia documental elaborada por terceros y retenida por la entidad:

\begin{tabular}{|c|c|c|c|c|c|c|c|}
\hline \multicolumn{3}{|c|}{ POCO FRECUENTE } & \multicolumn{2}{|l|}{ Probabilidad 1} & \multicolumn{2}{|c|}{ BAJO } & Nivel de riesgo 1 \\
\hline \multicolumn{3}{|c|}{ FRECUENTE } & \multicolumn{2}{|l|}{ Probabilidad 2} & \multicolumn{2}{|c|}{ MODERADO } & Nivel de riesgo 2 \\
\hline \multicolumn{3}{|c|}{ MUY FRECUENTE } & \multicolumn{2}{|l|}{ Probabilidad 3} & \multicolumn{2}{|c|}{ ALTO } & Nivel de riesgo 3 \\
\hline \multicolumn{8}{|c|}{ Matriz De Valoración De Riesgos Del Auditor De La Obtención De La Evidencia Documental } \\
\hline $\begin{array}{c}\text { Tipo De } \\
\text { Evidencia }\end{array}$ & \multicolumn{2}{|c|}{ Tipo De Riesgo } & Descripción & $\mathrm{NIA}$ & Probabilidad & \begin{tabular}{|c|}
$\begin{array}{c}\text { Nivel De } \\
\text { Riesgo }\end{array}$ \\
\end{tabular} & Sugerencia \\
\hline \multirow{4}{*}{ 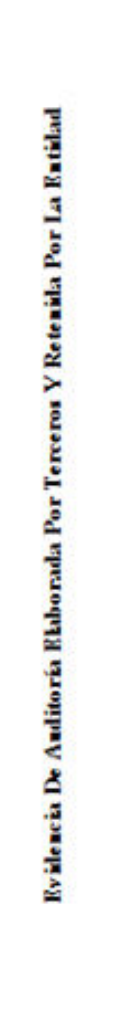 } & $\begin{array}{l}\text { Riesgo de } \\
\mathrm{la} \\
\text { Auditoria }\end{array}$ & $\begin{array}{l}\text { Confirmaciones } \\
\text { o Respuestas no } \\
\text { Suficientes }\end{array}$ & $\begin{array}{l}\text { Es presentado cuando los } \\
\text { cuestionarios aplicados } \\
\text { verbalmente o por escrito no } \\
\text { suministran datos relevantes para } \\
\text { la obtención de la evidencia. }\end{array}$ & 505 & Poco Frecuente & Bajo & $\begin{array}{l}\text { "Informar la situación a la } \\
\text { administración, para que se ordene a los } \\
\text { funcionarios que suministren la } \\
\text { información completa que requiere el } \\
\text { equipo auditor. }\end{array}$ \\
\hline & $\begin{array}{l}\text { Riesgo } \\
\text { del } \\
\text { Auditor }\end{array}$ & Defensa & $\begin{array}{l}\text { Se da cuando el auditor apoya una } \\
\text { posición hasta tal punto que su } \\
\text { objetividad pueda verse } \\
\text { comprometida. Ejemplo, cuando } \\
\text { el auditor actia en defensa del } \\
\text { ente auditado en un litigio o } \\
\text { disputa. }\end{array}$ & 260 & Poco Frecuente & Moderado & $\begin{array}{l}\text { * Ejecutar politicas y procedimientos que } \\
\text { prohiban a los auditores que asistan al } \\
\text { cliente de auditoria de tomar decisiones } \\
\text { gerenciales en nombre del cliente. }\end{array}$ \\
\hline & $\begin{array}{l}\text { Riesgo } \\
\text { Humano }\end{array}$ & $\begin{array}{l}\text { Manipulación o } \\
\text { Modificación de } \\
\text { Los Documentos }\end{array}$ & $\begin{array}{l}\text { Aparece al presentarse } \\
\text { modificación de la imformación } \\
\text { alterando los datos almacenados, } \\
\text { generando inseguridad en la } \\
\text { documentación presentada. }\end{array}$ & 230 & Poco Frecuente & Alto & $\begin{array}{l}\text { * Documentar detalladamente cualquier } \\
\text { novedad que conduzca a poner en riesgo } \\
\text { la seguridad de la información, posterior } \\
\text { a la revisión y comparación de los } \\
\text { registros del sistema con los soportes en } \\
\text { fisico para asi amalizar la situación y crear } \\
\text { omodificar controles en pro del } \\
\text { aseguramiento documental }\end{array}$ \\
\hline & Simiestro & $\begin{array}{l}\text { Daños en el } \\
\text { Sistema de } \\
\text { Información } \\
\text { Contable }\end{array}$ & $\begin{array}{l}\text { Por mal manejo o por condiciones } \\
\text { informaticas se puede producir un } \\
\text { software malicioso que puede } \\
\text { dañar el equipo provocando el } \\
\text { borrado de archivos, registros } \\
\text { contables o incluso del sistema } \\
\text { operativo al completo. }\end{array}$ & 530 & Poco Frecuente & Moderado & $\begin{array}{l}\text { * Para prevenir infecciones de virus } \\
\text { mformático, los usuarios, no deben hacer } \\
\text { uso de software que no hayan sido } \\
\text { proporcionados y validados. De igual } \\
\text { manera se debe capacitar a los } \\
\text { funcionarios para darle adecuado manejo } \\
\text { al sistema de información. }\end{array}$ \\
\hline
\end{tabular}

Fuente: Elaboración propia.

La anterior matriz, muestra que,en la ejecución de sus labores, el auditor debe aplicar procedimientos que le permitan identificar y valorar los riesgos de que existan errores o momento deobtenerevidencia documental suficiente, relevante y apropiada para poder basar su opinión y emitir un informe de auditoría. De igual manera, se puedeapreciar que la calidad de la evidencia está influida por su fuente de procedencia, es decir, las que son independientes 
de la empresa, em principio, ofrecen mayores garantías que las que están bajo el control de la misma; la evidencia obtenida directamentepor el auditor es más persuasiva que la que procede de un tercero o de la propia empresa.; y cuando la evidencia de una pruebase corrobora con la de otras, la confianza es superior.

\subsection{PROCEDIMIENTO PARA LA OTENCIÓN, ANÁLISIS Y PRESENTACIÓN DE LA EVIDENCIA DOCUMENTAL}

Además de otras fuentes internas o externas a la entidad, los registros contables de la entidad son una fuente importante de evidencia de auditoría. Así mismo, la información que se utiliza como evidencia de auditoría puede haberse preparado utilizando el trabajo de un experto de la dirección. La evidencia de auditoría comprende tanto la información que sustenta y corrobora las afirmaciones de la dirección como cualquier información que contradiga dichas afirmaciones. Adicionalmente, en algunos casos, el auditor utiliza la ausencia de información (por ejemplo, la negativa de la dirección a realizar una manifestación que se le haya solicitado) y, en consecuencia, constituye también evidencia de auditoría.

La mayor parte del trabajo del auditor consiste en obtener y evaluarla evidencia documental de auditoría con el fin de poder formarse una opinión y emitir su informe. Para ello deben aplicarse procedimientos de auditoría, los cuales pueden incluir la inspección, la observación, la confirmación, el cálculo, y los procedimientos analíticos, a menudo combinados entre sí, además de la indagación. Aunque la indagación puede proporcionar evidencia de auditoría importante, e incluso puede proporcionar evidencia de una incorrección, normalmente no proporciona, por sí sola, evidencia de auditoría suficiente sobre la ausencia de una incorrección material en las afirmaciones, ni sobre la eficacia operativa de los controles(NIA, 2013).

Acontinuaciónse presenta un diagrama de procesos, que refleja los procedimientos, métodos y técnicas a utilizar para la obtención, análisis y presentación de la evidencia documental en el encargo de auditoría. 
Figura 3. Diagrama de procesos para la obtención, análisis y presentación de la evidencia documental:

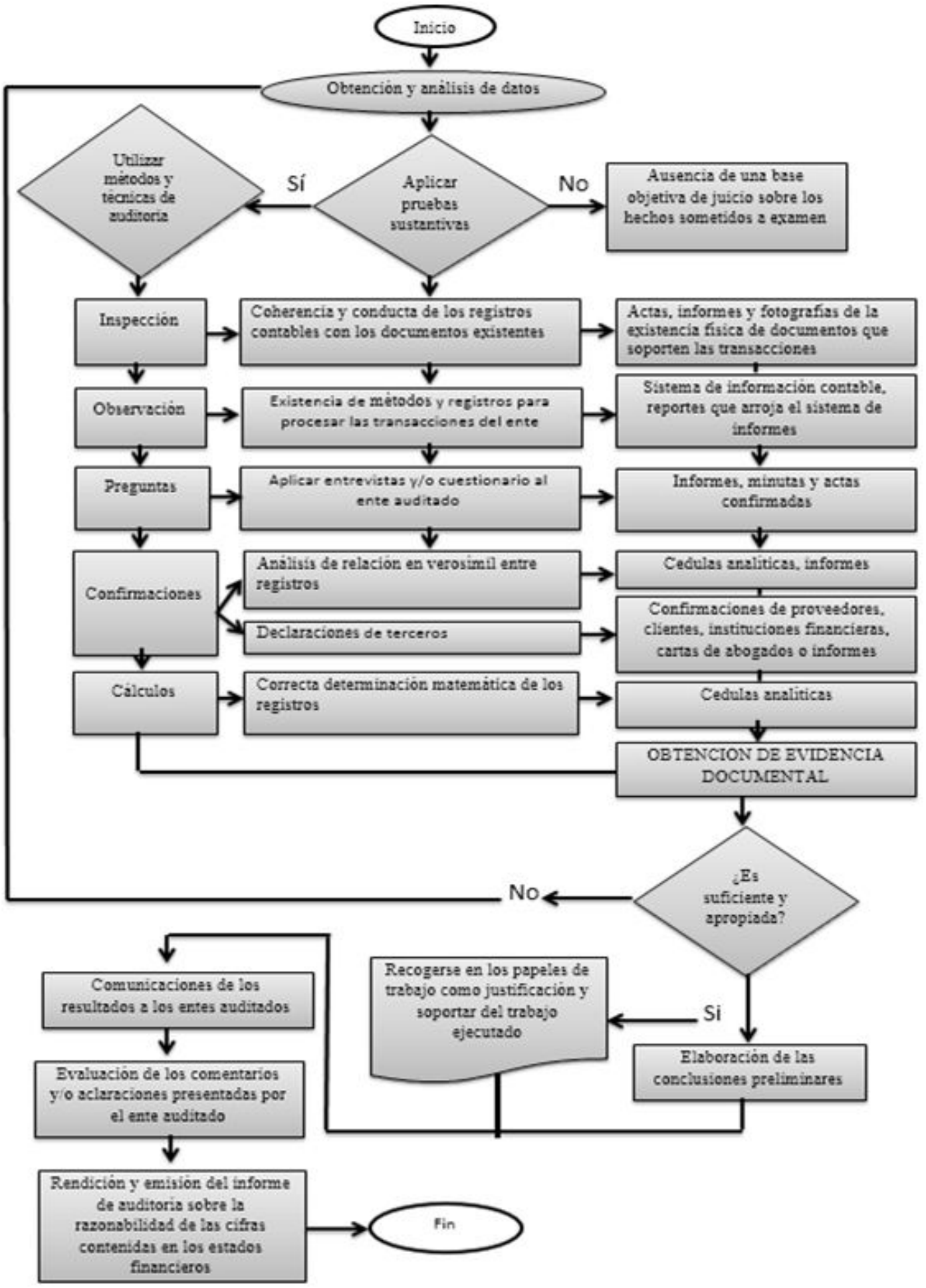

Fuente: Elaboración propia. 


\subsection{LA EVIDENCIA DOCUMENTAL DEL PERITO CONTABLE EN EL PROCESO JUDICIAL}

A nivel general es deber de toda persona, denunciar ante la Fiscalía la comisión de un hecho que pueda ser considerado como delito; y es deber de la Fiscalía investigar, perseguir y acusar a los presuntos responsables de su comisión.Por esta razón, desde el año 2004, la Fiscalía General de la Nación ha hecho énfasis en el grado de importancia que debe brindarse al manejo de las pruebas recolectadas por el auditor en el proceso de investigación en el encargo de auditoría. En consecuencia, el auditor debe tener un amplio conocimiento de las diferentes maneras que existen para cometer delitos económicos o financieros que pueden suceder en el ejercicio de la actividad económica de las organizaciones. Así las cosas, las pruebas periciales deben cumplir con los requerimientos establecidos por la ley, para lo cual el auditor debe aplicar diferentes técnicas, procedimientos y utilizar instrumentos que propendan hacia la legalidad de la prueba. (Plazas, Hernández, \& Florez, 2016)

En efecto, la ley 906 de 2004 (Congreso de la Republica de Colombia, 2004) señala que para demostrar la autenticidad de los elementos materiales probatorios y la evidencia física, se dará uso a un proceso que garantice la veracidad de la información recolectada cadena de custodia- por ser un sistema documentado, formado por una serie de instrucciones y registros, que se aplica a los documentos probatorios y a la evidencia documental por parte de las personas responsables de su manejo. A su vez, el código de procesamiento civil en Colombia(Decreto 1400, 1970) expone que, para proferir una decisión de fondo, la regla general consiste en que esta debe fundamentarse en pruebas regular y oportunamente allegadas al proceso. A partir de ahí, el debido proceso, establece que lo más importante dentro del trabajo del perito contable, es conocer el valor de la evidencia suficiente y competente, en aras de justificar lo que se quiere probar.

Bajo este referente, el auditor, como experto contable deberá presentar su evidencia documental en el proceso judicial y tener a la mano las pruebas para que se demuestre la realidad de los hechos, teniendo en cuenta la responsabilidad, la seguridad y la preservaciónde cada una de las evidencias halladas durante la ejecución del trabajo. 


\section{ESTUDIO DE CASO}

\subsection{ANTECEDENTES}

Una entidad de carácter público (Alcaldía Municipal), ubicada en el Norte del departamento del Tolima realizó transición hacia cambio de gobierno en el año 2016. La situación se deriva de los hechos ocurridos el 26 de junio del mismo año cuando una empresa contratista presentó el balance financiero en el acta de liquidación; sin embargo, el actual Secretario de Hacienda del municipio al verificar los giros realizados a la Sociedad durante las vigencias anteriores, determinó que el giro presupuestal de gastos del 26 de junio por un valor de 65 millones de pesos, no se encontraba relacionado en el acta de liquidación. Por ende, el presidente de la Sociedad corroboró la información al no tener registro de dicho giro en ninguna de las cuentas bancarias adscritas al convenio, el cual tenía por objeto la construcción de un puente colgante

La entidad pública inició procesos de conciliación financiera, revisión de giros y pagos realizados en los últimos años, encontrando con ello que sehabíaefectuadoun pago por el valor solicitado, pero a la empresa contratista nunca ingresó el mismo.En efecto, el actual secretario de hacien da procedió a verificar las transacciones por medio de la banca virtual y allí encontró un giro hecho en junio de 2015 desde la cuenta oficial por la cifra anteriormente citada y el mismo día se registró una cantidad igual, en la cuenta personal del exsecretario de Hacienda. Con este hallazgo se procedió a entablar las acciones judiciales correspondientes.

\subsection{PROCEDIMIENTO}

La situación anteriormente descrita sirvió para que se empezara a revisar la contabilidad, razón por la que la Alcaldía Municipal contrató un perito contable que se encargara de la inspecciónde documentos y soportes contables corroborando con las transacciones electrónicas que arrojaba el sistema. Con ello se determinó que la cuenta conciliaba porque efectivamente el giro presupuestal de gastos había sido afectado por la misma cuantía que se encontraba registrada en el sistema de información contable como egreso. Sin embargo, el perito sugirió a la administración que se consultara a la respectiva entidad financiera, quien, mediante oficio del 30 de junio de 2016 de la Directora del banco, expresó que el día 26 de junio de 2015, de una cuenta de propiedad del municipio, se hizo una 
transacción de 65 millones de pesos a una cuenta de ahorros cuyo titular era el ex secretario de hacienda.

De igual manera, la directora del banco manifestó que la plataforma de la banca virtual es de manejo dual, es decir, que tanto el exsecretario de Hacienda como el exmandatario tenían que digitar una clave para que el banco realizara el giro de los recursos. Con esto, quedó claro que no se trató de un caso aislado, sino de acciones recurrentes entre los dos exfuncionarios.

En consecuencia, el alcalde interpuso la denuncia a la Fiscalía General de la Nación, la Contraloría General de la Repúblicay la Procuraduría. Una vez conocida la situación, la Fiscalía General envió un grupo de funcionarios hasta al municipio para realizar auditoria, proceso en el cual se exigierondocumentos, soportes y estados de cuenta para validar la información. El equipo auditor se llevó consigo la copia de los soportes para iniciar las respectivas investigacionesy presentaron requerimiento ante un Juez de la República para que solicitara las pruebas documentales a la entidad financiera y así verificar las transacciones,encontrándose con ello que al ex secretario de hacienda se le habría consignado en su cuenta personal $\$ 878$ millones del Estado, como también se descubrieron consignaciones a la cuenta de la esposa del exsecretario y hasta al mismo exalcalde, siendo cerca de ochenta movimientos bancarios que representarían $\$ 1.304$ millones de pesos.

Después de 30 díasla Fiscalía General de la Nación, continuó en proceso investigativo se recaudó más evidencia y fortalecieron el material probatorio, con lo cualse realizó la acusac ión yuna vez legalizado el proceso de detención, se adelantó la audiencia de imputación de car gos, espacio en el que un Juez con función de Control de Garantías determinó medida de aseg uramiento intramural en establecimiento carcelario contra del exsecretario de hacienda del mu nicipio, por su presunta participación en corrupción administrativa. Servidores del cuerpo técn ico de investigación capturaron por orden judicial,al ex funcionario, quien debería responder $\mathrm{p}$ or los delitos de peculado por apropiación y falsedad ideológica en documento público.

Tras suscribir un preacuerdo con la Fiscalía General de la Nación, el exsecretario de H acienda, devolvió una millonaria suma de dinero, como una forma de resarcir los daños causa dos a la administración local.En definitiva, la evidencia documental obtenida, analizada y pres entada por el perito contable ante los entes de control en el proceso judicialse considera bastan te significativa, y de esta forma se puede asegurar que la etapa de ejecución de la auditoría cu 
enta con los elementos necesarios para el levantamiento de una prueba amplia y suficiente del delito financiero que se cometió en esta entidad estatal.

Tabla 4. Matriz de valoración de riesgos en la obtención de evidencia documental para el caso de estudio:

\begin{tabular}{|c|c|c|c|c|c|}
\hline \multicolumn{2}{|c|}{ BAJO } & Nivel de riesgo 1 & & & \\
\hline \multicolumn{2}{|c|}{ MODERADO } & Nivel de riesgo 2 & & & \\
\hline \multicolumn{2}{|c|}{ ALTO } & Nivel de riesgo 3 & & & \\
\hline \multicolumn{6}{|c|}{$\begin{array}{c}\text { MATRIZ DE VALORACION DE RIESGOS EN LA OBTENCION DE LA EVIDENCIA DOCUMENTAL PARA EL CASO } \\
\text { DE ESTUDIO }\end{array}$} \\
\hline $\begin{array}{l}\text { TIPO DE } \\
\text { EVIDENCIA }\end{array}$ & DOCUMENTO & $\begin{array}{l}\text { DESCRIPCIÓN DEL } \\
\text { RIESGO }\end{array}$ & $\begin{array}{l}\text { NIVEL DE } \\
\text { RIESGO }\end{array}$ & NIA & RECOMENDACIÓNES \\
\hline $\begin{array}{l}\text { Evidencia de } \\
\text { Auditoría } \\
\text { Elaborada y } \\
\text { Retenida por } \\
\text { Terceros }\end{array}$ & $\begin{array}{c}\text { Comprobante de } \\
\text { consignación } \\
\text { bancaria }\end{array}$ & $\begin{array}{c}\text { Problemas para que la } \\
\text { entidad financiera } \\
\text { facilite la información } \\
\text { requerida respecto al } \\
\text { movimiento de las } \\
\text { cuentas }\end{array}$ & Moderado & 520 & \multirow{3}{*}{$\begin{array}{l}\text {-Intervención constante de } \\
\text { los entes de control en todos } \\
\text { los procesos que se lleven a } \\
\text { cabo dentro de la entidad. } \\
\text {-Los entes competentes sean } \\
\text { quienes designen y } \\
\text { contraten el personal de } \\
\text { control intemo de la entidad } \\
\text { pública con el fin de mitigar } \\
\text { las falencias y los riesgos } \\
\text { que se pueden presentar } \\
\text { tanto en el área contable } \\
\text { como administrativa para el } \\
\text { manejo de la } \\
\text { documentación. }\end{array}$} \\
\hline $\begin{array}{l}\text { Evidencia de } \\
\text { Auditoría } \\
\text { Elaborada por } \\
\text { Terceros y } \\
\text { Retenida por la } \\
\text { Entidad }\end{array}$ & $\begin{array}{l}\text { Extracto } \\
\text { bancario }\end{array}$ & $\begin{array}{c}\text { Los estados de cuenta } \\
\text { pueden extraviarse de } \\
\text { la entidad con el fin de } \\
\text { que no queden pruebas } \\
\text { de las transacciones } \\
\text { realizadas } \\
\end{array}$ & Alto & 230 & \\
\hline $\begin{array}{l}\text { Evidencia de } \\
\text { Auditoría } \\
\text { Elaborada y } \\
\text { Retenida por la } \\
\text { Entidad }\end{array}$ & $\begin{array}{c}\text { Soporte del giro } \\
\text { presupuestal de } \\
\text { gastos y } \\
\text { conciliación } \\
\text { bancaria }\end{array}$ & $\begin{array}{c}\text { Manipulación o } \\
\text { modificación de la } \\
\text { información por parte } \\
\text { de los funcionarios }\end{array}$ & Alto & 200 y 315 & \\
\hline
\end{tabular}

Fuente: Elaboración propia.

Como se puede apreciar en la matriz del caso de estudio, los riesgos presentados durante e 1 proceso de obtención, análisis y presentación de la evidencia documental fueron mitigados, $\mathrm{p}$ uesto que la entidad financiera emitió la información necesaria para comprobar el fraude, y lo s soportes documentales fueron encontrados dentro del archivo de la entidad sin haber sido m anipulados o modificados por funcionarios cómplices del delito cometido.

\section{CONCLUSIONES}

En el artículo se analiza la importancia que tiene en el encargo de una auditoría la práctica de la evidencia documental a través de la aplicación de técnicas y procedimientos, así como también la valoración e identificación de los riesgos, lo cual se evidencia en el 
desarrollo del documento y por lo que se concluye que cuando la evidencia documental es suficiente y relevante, efectivamente disminuye los riesgos en el proceso de la auditoría, ya que permite obtener pruebas de mucha validez legal sobre las cuales el auditor puede basar su opinión y emitir un informe que contenga un alto grado de confianza, tanto para quien contrata la auditoría, como para que sea tenido en cuenta en un proceso judicial.

Con base en lo anterior, al haber sido ejercida la auditoría y tenida en cuenta la evidencia documental — en este estudio de caso específico - por un perito contable y por los entes de control gubernamental, se puede comprobar que su obtención, análisis y presentación propende por la disminución de riesgos en el proceso y que este tipo de evidencia es uno de los tipos de evidencia más importantes que utiliza el auditor, y en el cual encuentra una base de juicio razonable dependiendo de la confianza que le ofrezca el sistema de control interno de la empresa.

Finalmente, cuando el auditor recopila prueba amplia y suficiente que responde a las necesidades de los usuarios de la información, y se apoya en instrumentos tradicionales y especializados, la evidencia documental se toma como prueba fidedigna y confiable y se reviste de gran importancia en el encargo de auditoría, dado a que genera alto nivel de confianza y a su vez ofrece mayores garantías porque puede ser usada ante los tribunales y se le da la categoría de prueba pericial.

\section{REFERENCIAS BIBLIOGRÁFICAS}

NIIC 1. (15 de Diciembre de 2009). Norma Internacional de Control de Calidad 1. Obtenido de https://www.facpce.org.ar/web2011/files/proyectos_rt/anexo_prt26.pdf

2015, D. 2. (14 de 12 de 2015). MINCIT. Obtenido de MINCIT: http://www.mincit.gov.co/loader.php?IServicio=Documentos\&lFuncion=verPdf\&id=79561\&n ame=DECRETO_2420_DE_2015.pdf\&prefijo=file

31000:2009, I. G. ((s.f)). International Organization For Standardization. Obtenido de International Organization For Standardization: https://www.iso.org/iso-31000-riskmanagement.html

500, N.-E. (15 de 10 de 2013). Evidencia de Auditoria . Obtenido de Evidencia de Auditoria : http://www.icac.meh.es/NIAS/NIA\%20500\%20p\%20def.pdf

Bautista, J. N. (02 de Noviembre de 2013). Jonlob. Obtenido de Jonlob: https://sites.google.com/site/jorgenlopezbautista/5 
Blanco, S. A. (abril de 2010). issuu. Obtenido de https://issuu.com/samantilla/docs/que_es_aseguramiento

Carvajal., A. P. ((s.f)). El Riesgo Empresarial Y Su Relacion Con Las Normas Internacionales De Auditoria Y Aseguramiento. Obtenido de El Riesgo Empresarial Y Su Relacion Con Las Normas Internacionales De Auditoria Y Aseguramiento: file://C:/Users/Yeimy/Downloads/267-978-2-PB\%20(1).pdf

Commission, C. O. ((s.f)). Coso Committee Of Sponsoring Organizations Of The Treadway Commission. Obtenido de Coso Committee Of Sponsoring Organizations Of The Treadway Commission: https://www.coso.org/Pages/default.aspx

Congreso de la Republica de Colombia. (2004). Ley 906 de 2004. Obtenido de http://www.alcaldiabogota.gov.co/sisjur/normas/Norma1.jsp?i=14787

Contabilidad, F. I. (2007). Normas Internacionales De Auditoria (9a ed). Mexico D.F: Instituto Mexicano De Contadores Publicos .

Cruz Navas et al., 2. (Noviembre de 2011). Universidad Nacional Experimental De Los Llanos Occidentales. Obtenido de Universidad Nacional Experimental De Los Llanos Occidentales: http://www.monografias.com/trabajos96/documentos/documentos.shtml

Decreto 1400. (6 de Agosto de 1970). Decreto 1400 de 1970.Obtenido de http://www.alcaldiabogota.gov.co/sisjur/normas/Norma1.jsp?i=6923

Dora Marcela Rodríguez García et al,. (2016). La auditoría y su control de calidad: una mirada desde las normas de aseguramiento de la información, en Colombia. Contexto-Revista de Investigaciones//ISSN 2339-3084, 5. Obtenido de universidad la gran colombia.

Ferrer, H. V. (12 de Abril de 2012). Vicont-Asesorias- Auditores-Revisorias . Obtenido de Vicont- Asesorias- Auditores- Revisorias : http://vikonta.blogspot.com.co/2012/04/laevidencia-en-auditoria.html

Figueroa, V. M. (2009). Tec Empresarial . Obtenido de Tec Empresarial: http://revistas.tec.ac.cr/index.php/tec_empresarial/article/viewFile/657/584

Glezen, T. (1997). Auditoria Iintegracion de Conceptos y Procedimientos. Colombia: Limusa, S.A.

Gómez, E. H. (18 de Abril de 2006 ). gestiopolis. Obtenido de gestiopolis: https://www.gestiopolis.com/evidencias-papeles-trabajo-auditoria/

IBEF. (2012). Instituto Barcelona De Estudios Financieros - IBEF. Obtenido de Instituto Barcelona De Estudios Financieros: http://aulavirtual.campusibef.es/webibef/modprint2.aspx $?$ doc $=685739 \& a=0$

IFAC. (15 de Diciembre de 2009). International Standard On Auditing 240. Obtenido de International Standard On Auditing 240: http://www.ifac.org/system/files/downloads/a012- 
2010-iaasb-handbook-isa-240.pdf

Instituto Barcelona De Estudias Financieros. (2012). Obtenido de Instituto Barcelona De Estudios

Financieros:

http://aulavirtual.campusibef.es/webibef/modprint2.aspx?doc $=685739 \& a=0$

Leuro-Carvajal, A. P. (noviembre de 2014). researchgate.net. Obtenido de https://www.researchgate.net/publication/314177334_El_riesgo_empresarial_y_su_relacion_c on_las_Normas_Internacionales_de_Auditoria_y_Aseguramiento

Luis Guillermo Plata Paez et al., . (13 de Julio de 2009). Alcaldia De Bogota. Obtenido de Alcaldia De Bogota: http://www.alcaldiabogota.gov.co/sisjur/normas/Norma1.jsp?i=36833

Luna, Y. B. (2004). Manual De Auditoria Y De Revisoria Fiscal. Bogota : Esfera Editores LTDA.

Martinez, M. H. (05 de Julio de 2016). Auditool-Red Global de Conocimientos en Auditoria y Control Interno. Obtenido de https://www.auditool.org/blog/auditoria-externa/772-laevidencia-de-auditoria

Medina, F. M. (26 de Mayo de 2014). Centro Contable Consultores \& Asesores. Obtenido de Centro Contable Consultores \& Asesores: http://centrocontable.com.co/index.php/blog/noticias-de-interes/actualidad/item/53-nia500\#.WUBwBWg1_IV

Normas Relativas a la Ejecucion del Trabajo. (2003). Fccea.unicauca.edu.co. Obtenido de Fccea.unicauca.edu.co: http://fccea.unicauca.edu.co/old/tgarf/tgarfse33.html

Plazas, R. A., Hernández, L., \& Florez, M. H. (15 de Noviembre de 2016). Ediciones Iusta. Obtenido de revistas.usta.edu.co/index.php/iusta/article/view/3525

Pombo, J. R. (2013). Contabilidad Y Fiscalidad . Paraninfo.

Rodriguez, B. (29 de Mayo de 2015). Evidencia en Auditoría. Obtenido de https://es.slideshare.net/balbinoantoniorg/evidencia-en-auditoria

Rodriguez, J. G. (16 de Junio de 2011). Codigo de Comercio . Bogota, Provincia, Region: Legis. Obtenido de Diario Oficial 33.339: http://www.alcaldiabogota.gov.co/sisjur/normas/Norma1.jsp?i=41102

Sandovalesco. (2013). Financiera Sandovalesco . Obtenido de Financiera Sandovalesco : http://www.fisan.com.mx/srvesaudin.html

Sandovalesco, F. (2013). Fisan. Obtenido de FISAN: http://www.fisan.com.mx/srvesaudin.html

Trujillo, C. G. (13 de Diciembre de 1990). Mineducacion. Obtenido de Mineducacion: http://www.mineducacion.gov.co/1759/articles-104547_archivo_pdf.pdf 
Vesper, J. (2009). Buenas Practicas De La Documentacion . Obtenido de Buenas Practicas De La Documentacion : https://bpl2009.wikispaces.com/file/view/BPdocumentacion.pdf

Yepes, J. L. (1993). ¿Que Es Documentacion? Madrid : Sintesis .

Yucra, L. (7 de 10 de 2012). Auditoria Administrativa . Obtenido de Auditoria Administrativa : http://auditoriaadministrativadued.blogspot.com.co/2012/10/elaboracion-del-informe-deauditoria.html

Zambrana, G. C. (8 de 02 de 2012). Evidencia Documental . Obtenido de Evidencia Documental : http://www.mailxmail.com/evidencia-documental-auditoria_h 